\title{
Late relapsing germ cell tumors with elevated tumor markers
}

\author{
Yue Che ${ }^{1}$. Achim Lusch ${ }^{1} \cdot$ Christian Winter $^{1} \cdot$ Robert Große Siemer $^{1,2} \cdot$ Carolin Buddensieck $^{1} \cdot$ Peter Albers ${ }^{1}$. \\ Andreas Hiester ${ }^{1}$ (1)
}

Received: 21 May 2021 / Accepted: 2 September 2021 / Published online: 13 September 2021

(C) The Author(s) 2021

\begin{abstract}
Purpose Late relapsing germ cell tumors (LR-GCT) are considered a rare distinct biologic entity as their clinical presentation and response to treatment is different to early recurrences. While serum tumor markers (AFP and B-HCG) play an important role at the time of first diagnosis to correctly classify prognosis and treatment of germ cell tumors, they may not have the same significance in a late relapse situation.

Patients and methods Thirty-seven patients with LR-GCT with elevated serum tumor markers were identified in our database. Twenty-six patients underwent primary surgical resection of the late relapsing tumor. Eleven patients received salvage chemotherapy and a post-chemotherapy residual tumor resection. Serum tumor markers, histological findings and oncological outcome were analyzed.

Results In the histopathological specimen, viable cancer was found in 20 cases (54\%) and teratoma was found in 16 cases (43\%). In nine cases (24\%), a somatic-type malignant transformation was present. In 19 of 37 patients (51.4\%), the late relapse specimen presented a histological type of GCT, which was not present in the primary histology. Twenty-two patients (59.5\%) were included in follow-up analysis. Mean and median follow-up time was 62.2 and 53 months, respectively. Seventeen patients (77.3\%) suffered a relapse or had progressive disease after LR therapy. Five patients $(22.7 \%)$ have been relapse-free after LR therapy (mean FU 61.6 months). Ten patients died of disease during follow-up (45.5\%) and had a mean time from LR to death of 66.4 months. Eleven patients were alive at last follow-up (mean FU 62.2 months). Relapse and survival rate were similar between patients who received primary resection of LR tumor and patients who received salvage chemotherapy followed by surgery.

Conclusion Patients with a late relapsing germ cell tumor and elevated markers have a poor prognosis and a high risk for another relapse independent on primary treatment. The histological type and aggressiveness of a late relapsing tumor cannot be predicted with serum tumor marker levels at the time of diagnosis of LR. In up to 54\% of cases, primary histology did not coincide with LR histology. Therefore, we propose primary surgical resection of a late relapsing tumor if a complete resection is feasible in order to gain exact histology and tailor further treatment.
\end{abstract}

Keywords Germ cell tumor $\cdot$ Late relapse $\cdot$ Surgery $\cdot$ Tumor marker

\section{Introduction}

In germ cell tumors (GCT), a tumor recurrence more than 2 years following initial treatment including chemotherapy is considered a late relapse (LR). It is a rare situation with a reported incidence of $3.2 \%$ in non-seminomatous GCT

Andreas Hiester

Andreas.Hiester@med.uni-duesseldorf.de

1 Department of Urology, Medical Faculty, Heinrich Heine University Düsseldorf, Moorenstr. 5, 40225 Düsseldorf, Germany

2 Department of Urology, Helios University Hospital Wuppertal, Wuppertal, Germany (NSGCT) and $1.4 \%$ in seminoma [1]. Late relapsing GCT (LR-GCT) are considered a distinct biologic entity, which is still not fully understood. Biologically, a LR-GCT is a tumor coming from an almost inactive or dormant state, which might have been triggered under cellular stress during cisplatin therapy. While resected specimens may contain 
all histological types of GCT [2], teratoma is the predominant one. This is related to the chemoresistant nature of teratomas.

Surgery has been proposed as the most important tool in the treatment of LR $[1,3,4]$ as LR tumors are often chemorefractory [5]. Because of the heterogeneous biology of LR-GCT, the general management recommendation is to completely resect the LR tumor or obtain a representative biopsy before salvage therapy is carried out.

In GCT, serum tumor markers (STM) play an important role at the time of first diagnosis to correctly classify the tumor and yield information about the prognosis [6]. Treatment is adjusted to marker levels, and markers are necessary to monitor treatment response. AFP is secreted by $40-60 \%$ of patients with embryonal cell carcinoma and yolk sac tumor, while HCG is elevated in $10-20 \%$ of patients with choriocarcinoma [7]. At the time of recurrence, GCT are frequently treated with salvage chemotherapy when STM are elevated. Only patients with marker-negative recurrence have an undoubtable indication for primary surgery. In late relapsing patients with elevated markers, the question of primary surgery or primary chemotherapy remains unsolved.

In this retrospective study, we aimed to investigate the significance of tumor marker elevation at the time of diagnosis of a LR. In this case series we analyzed 37 patients who had a LR with elevated tumor markers and underwent primary surgical resection of the tumor or residual tumor resection after chemotherapy.

\section{Patients and methods}

\section{Study population}

After institutional review board, patients underwent chemotherapy or primary surgical resection of a late relapsing germ cell tumor with elevated STMs. LR was defined as a relapse 2 years or later after last chemotherapy. Elevated STMs were defined by our laboratory as a level of human chorionic gonadotropin (B-HCG) equal to or greater than $2.0 \mathrm{mIU} / \mathrm{ml}$ and/or a level of alpha-fetoprotein (AFP) equal to or greater than $7.0 \mu \mathrm{g} / \mathrm{l}$. Patient characteristics are shown in Table 1.

\section{Statistical methods}

Means, medians and interquartile ranges were calculated for measured variables, respectively. Fisher's exact test or $t$ tests were performed as indicated in the results section. Statistical tests were performed using online tools provided by VassarStats. All tests were set with a significance level at $p$ value $<0.05$.

\section{Results}

Between July 2008 and December 2020, 671 retroperitoneal lymph-node dissections (RPLND) were performed in our referral center. We queried our database for patients who had a LR-GCT with elevated STMs. Thirty-seven patients were identified. Twenty-six patients underwent primary surgical resection of the LR. The decision for a surgical approach was discussed in the interdisciplinary tumor board with the primary goal of a complete resection of all lesions. Eleven patients received second-line or salvage chemotherapy and a post-chemotherapy residual tumor resection.

\section{Previous therapy, relapse location and time to relapse}

All patients had a NSGCT with a clinical stadium II (IS) or III at first diagnosis. Four patients underwent primary RPLND with adjuvant chemotherapy with two cycles of bleomycin, etoposide and cisplatin (BEP). Thirty-two patients received standard first-line chemotherapy, and one patient received primary high-dose chemotherapy at diagnosis (Fig. 1). Twenty-eight patients had a LR with elevated STMs after first-line therapy. Of these 28 patients, eight patients received second-line chemotherapy with subsequent postchemotherapy residual tumor resection (PC-RTR). Twenty patients underwent primary resection for LR tumor.

Nine patients had an early relapse after first-line therapy and received second-line therapy. These nine patients had a LR with elevated STMs after second-line therapy. Three patients received third-line chemotherapy and residual tumor resection. Six patients underwent primary LR resection.

The most frequent site of a LR was the retroperitoneum (36/37, 97\%). Other relapse sites were the lung (4), the mediastinum (5), liver (2), bone (2) and supraclavicular (1) (Table 1).

Mean and median time from last therapy to LR was 114 and 96 months, respectively (med. IQR 62-127). The longest time to LR was 304 months ( 25 years).

\section{Serum tumor markers and histology (Table 2)}

All patients had elevated STM at the time of LR. AFP was elevated in 35 patients, and B-HCG was elevated in two patients. This unequal distribution can be explained by the main evidence of teratoma and yolk sac in the histological specimens.

Patients who received second-line or salvage chemotherapy had a mean AFP level of 20,328 $\mu \mathrm{g} / \mathrm{l}$ (IQR $139-33,000 \mu \mathrm{g} / \mathrm{l})$. Nine patients of this group still had 
Table 1 Patients' characteristics

\begin{tabular}{|c|c|c|c|}
\hline All patients characteristic & $n=$ & Primary LR section $n=$ & $\begin{array}{l}\text { Chemotherapy } \\
\text { with PC-RTR } \\
n=\end{array}$ \\
\hline
\end{tabular}

Primary histology

Seminoma

NSGCT

Ectragonadal

Yes

Initial clinical stage

IS

II

III

Initial IGCCG

Good

Intermediate

Poor

Unknown

Site of late recurrence

Retroperitoneal

Pulmonal

Mediastinum

Liver

Bone

Superaclavicular

Time from end of last treatment to relapse

Mean

Median

Range

Markers before chemotherapy

AFP positive, $n=$

$\operatorname{AFP}(\mu \mathrm{g} / \mathrm{l})$, mean

AFP median

AFP, range

AFP, IQR

HCG positive, $n=$

HCG (mlU/ml)

Markers before surgery

AFP positive, $n=$

$\operatorname{AFP}(\mu \mathrm{g} / \mathrm{l})$, mean

AFP median

AFP, range

AFP, IQR

HCG positive, $n=$

HCG (mlU/ml)

Histology

EC

Yolk sac

Choriocarcinoma

Teratoma

Seminoma

Somatic-type malignant transformation
0

37

26

11

5

4

1

1

5

5

17

14

12

5

16

11

2

5

8

8

3

6

6

2

2

36

25

11

4

5

2

2

1

114 months

96 months

30-304 months

0

4

0

0

1

120 months

101 months

30-304 months

4

1

2

2

0

99 months

96 months

54-160 months

10

20,328

16,357

31-50,907

139-33,000

1

180

25

492.25

38.45

1104

7.4-5480

138

12.8-135.25

2.6-7000

47.75-945.5

1

2.1

0

623

82

0

$15 \quad 9$

11

5
9

4year 
Table 1 (continued)

Fig. 1 Previous therapies before late relapse and late relapse therapy. Blue box: salvage chemotherapy + PC-RTR, Green box: primary resection, $\mathrm{PEB}$ : cisplatin, etoposide, bleomycin, PEI: cisplatin, etoposide, ifosfamide, PE: cisplatin, etoposide, HDCT: high-dose chemotherapy, $\mathrm{m}+$ : serum marker positive, y.: years, CS: clinical stage

\begin{tabular}{|c|c|c|c|}
\hline All patients characteristic & $n=$ & Primary LR section $n=$ & $\begin{array}{l}\text { Chemotherapy } \\
\text { with PC-RTR } \\
n=\end{array}$ \\
\hline
\end{tabular}

Follow-up after late relapse

Incomplete follow-up data $\quad 14$

Relapse after late relapse $\quad 17$

Dead 10

Survived (follow-up $>2$ years) $\quad 11$

Follow-up time, mean 59 months

Follow-up time, median 49 months

NSGCT non-seminomatous germ cell tumor, $H C G$ human chorionic gonadotropin, $A F P$ alpha-fetoprotein

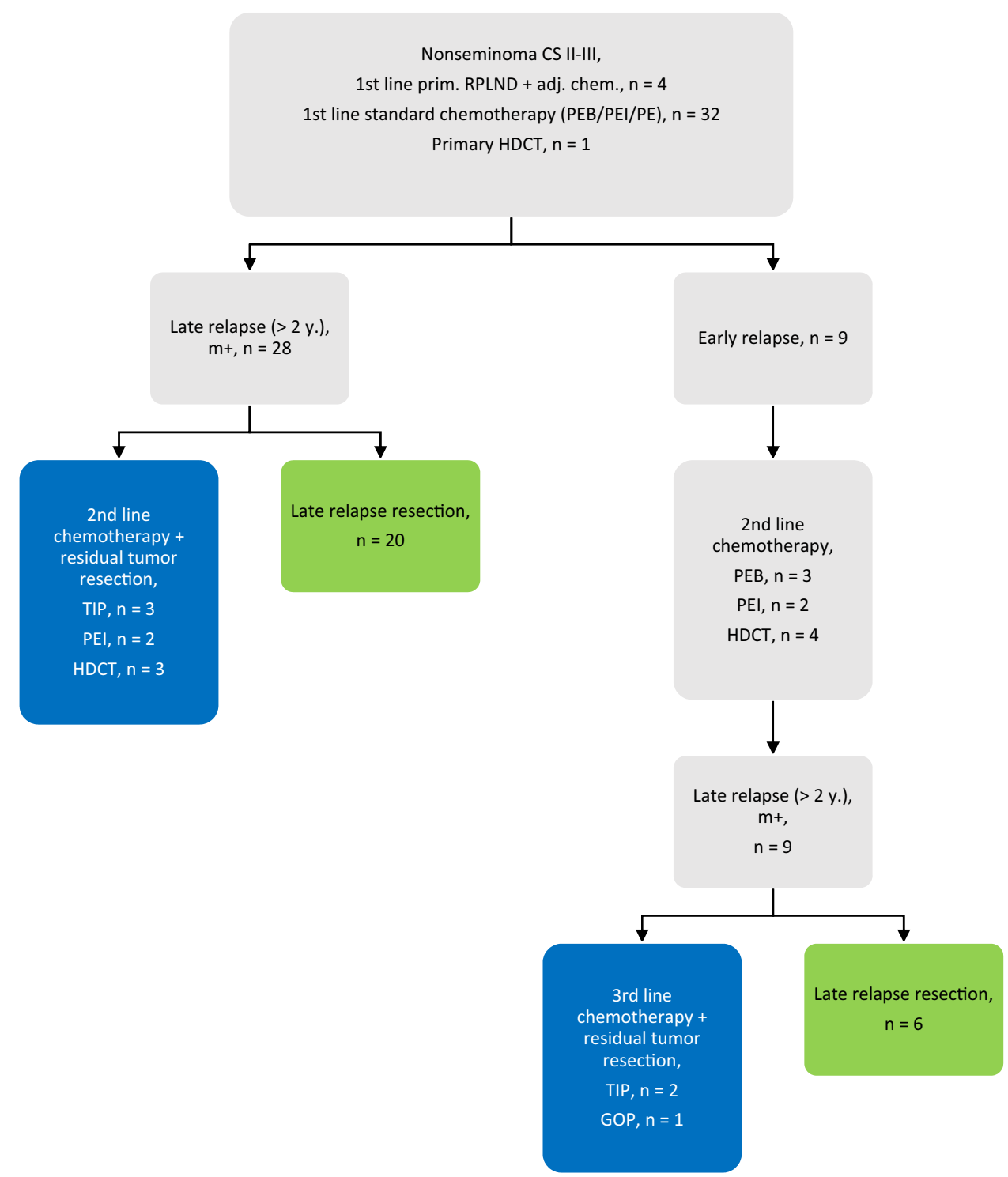

elevated STMs before PC-RTR with a mean AFP level of $1104 \mu \mathrm{g} / \mathrm{l}$ (IQR 48-946 $\mu \mathrm{g} / \mathrm{l})$. Patients who received primary surgery had a mean AFP level of $493 \mu \mathrm{g} / \mathrm{l}$ (IQR 13-135 $\mu \mathrm{g} / \mathrm{l})$ at LR. AFP level was significantly higher in patients who received primary chemotherapy than in patients who underwent primary surgery $(p=<0.0001)$. 
Table 2 Histology and tumor markers before surgery

\begin{tabular}{|c|c|c|c|c|c|c|c|c|c|}
\hline \multirow[b]{2}{*}{$\begin{array}{l}\text { Pat. } \\
\text { nr. }\end{array}$} & \multicolumn{7}{|c|}{ Exact Histology/Percentage } & \multirow{2}{*}{$\begin{array}{c}\text { AFP before } \\
\text { surgery } \\
\\
{[\mu \mathrm{g} / \mathrm{l}]}\end{array}$} & \multirow{2}{*}{$\begin{array}{c}\begin{array}{c}\text { HCG before } \\
\text { surgery }\end{array} \\
{[\mathrm{mU} / \mathrm{ml}]}\end{array}$} \\
\hline & EC & YS & CC & Necrosis & $\begin{array}{c}\text { Teratom } \\
\mathbf{a}\end{array}$ & $\begin{array}{c}\text { Semino } \\
\text { ma }\end{array}$ & $\begin{array}{c}\text { SMT } \\
0=\text { no } \\
1 \text { = yes }\end{array}$ & & \\
\hline 1 & 0 & 0 & 0 & 0 & 100 & 0 & 0 & 11.9 & 0.1 \\
\hline 2 & 50 & 0 & 0 & 50 & 0 & 0 & 0 & 2.5 & 2.1 \\
\hline 3 & 0 & 0 & 0 & 0 & 100 & 0 & 0 & 45 & 0.1 \\
\hline 4 & 0 & 70 & 0 & 0 & 30 & 0 & 0 & 45 & 0.1 \\
\hline 5 & 0 & 0 & 0 & 30 & 70 & 0 & 0 & 10.3 & 0.5 \\
\hline 6 & 10 & 0 & 0 & 90 & 0 & 0 & 1 & 7.4 & 0.1 \\
\hline 7 & 0 & 20 & 0 & 80 & 0 & 0 & 0 & 25 & neg. \\
\hline 8 & 0 & 0 & 0 & 50 & 50 & 0 & 0 & 9.9 & neg. \\
\hline 9 & 0 & 0 & 0 & 85 & 0 & 15 & 0 & 8 & 0.1 \\
\hline 10 & 0 & 0 & 0 & 30 & 70 & 0 & 1 & 16.1 & 0.2 \\
\hline 11 & 0 & 100 & 0 & 0 & 0 & 0 & 0 & 117 & 0.1 \\
\hline 12 & 100 & 0 & 0 & 0 & 0 & 0 & 0 & 190 & neg. \\
\hline 13 & 0 & 100 & 0 & 0 & 0 & 0 & 0 & 50 & neg. \\
\hline 14 & pos. & pos. & 0 & 1 & pos. & 0 & 0 & 448 & 0.1 \\
\hline 15 & 0 & 100 & 0 & 0 & 0 & 0 & 0 & 4000 & neg. \\
\hline 16 & 0 & 0 & 0 & 0 & 100 & 0 & 1 & 281 & neg. \\
\hline 17 & 85 & 0 & 0 & 15 & 0 & 0 & 0 & 27 & 0.7 \\
\hline 18 & 15 & 0 & 0 & 70 & 15 & 0 & 0 & 47.7 & 0.1 \\
\hline 19 & 0 & 30 & 0 & 15 & 55 & 0 & 0 & 5480 & 0.1 \\
\hline 20 & 0 & 0 & 0 & 70 & 30 & 0 & 0 & 11.8 & 0.1 \\
\hline 21 & 0 & 0 & 0 & 5 & 95 & 0 & 0 & 16 & 0,1 \\
\hline 22 & 0 & 40 & 0 & 0 & 60 & 0 & 0 & 844 & 0.3 \\
\hline 23 & 0 & 90 & 0 & 10 & 0 & 0 & 0 & 1581 & 0.4 \\
\hline 24 & 0 & 0 & 0 & 10 & 90 & 0 & 0 & 13.1 & 0.1 \\
\hline 25 & 0 & 0 & 0 & 20 & 0 & 0 & $1(80 \%)$ & 78 & 0.1 \\
\hline 26 & 0 & 0 & 0 & 2 & 80 & & $1(28 \%)$ & 31.9 & 0.1 \\
\hline 27 & 0 & 55 & 0 & 0 & 0 & 45 & 0 & 7000 & neg. \\
\hline 28 & 0 & 0 & 0 & 10 & 90 & 0 & 0 & 2533 & 0.2 \\
\hline 29 & 0 & 25 & 0 & 60 & 15 & 0 & 0 & 2.6 & 0.1 \\
\hline 30 & 5 & 0 & 0 & 90 & 5 & 0 & 0 & 120 & 0.1 \\
\hline 31 & 0 & 0 & 0 & 95 & 5 & 0 & 0 & 4 & 0.1 \\
\hline 32 & 0 & 0 & 0 & pos. & pos. & 0 & 1 & 138 & 0.1 \\
\hline 33 & 0 & 0 & 0 & 70 & 30 & 0 & 1 & 46.5 & 0.1 \\
\hline 34 & 35 & 0 & 0 & 50 & 15 & 0 & 0 & 400 & 0.1 \\
\hline 35 & 10 & 0 & 0 & 90 & 0 & 0 & 0 & 1491 & 1.3 \\
\hline 36 & 0 & 0 & 0 & 25 & 75 & 0 & 1 & 365 & 0.1 \\
\hline 37 & 0 & 0 & 0 & 0 & 100 & 0 & 1 & 49 & neg. \\
\hline
\end{tabular}

Primary LR resection group: green. LR chemotherapy with PC RTR group: blue. EC embryonal carcinoma, $Y S$ yolk sac, $C C$ choriocarcinoma, SMT somatic-type malignant transformation 
In the histopathological specimen, viable cancer was found in 20 cases $(54 \%)$ and teratoma was found in 16 cases (43\%). In 9 cases (24\%), a somatic-type malignant transformation was present. The exact histological findings are shown in Table 2.

Looking closer at the preoperative STM levels, specimens containing yolk sac tumor had significantly higher AFP levels (mean AFP level $=1801 \mu \mathrm{g} / \mathrm{l}$ ) than specimens containing embryonal carcinoma (mean AFP level $303 \mu \mathrm{g} / 1$, one-tailed $t$ test, $p=0.0412$ ) or teratoma (mean AFP level $224 \mu \mathrm{g} / \mathrm{l}$, one-tailed t test, $p=0.035)$.

In 19 of 37 patients (51\%), the LR specimen presented a histological type of GCT, which was not present in the primary histology. In four patients, the primary histology was only known as NSGCT without any further specification (Table 3).

\section{Follow-up and outcome}

Fifteen patients (41\%) were lost to follow-up (FU) or had insufficient follow-up time ( $<2$ years) to be included in follow-up analysis. Twenty-two patients $(60 \%)$ were included in follow-up analysis. Mean and median FU time was 62 and 53 months, respectively. Relapse and survival rate were similar in patients who received primary resection of LR tumor and patients who received salvage chemotherapy (Table 4). The differences between therapy groups were not statistically significant (Fisher test, $p=0.66$ and 1, respectively). Seventeen patients $(77 \%)$ suffered a relapse or had progressive disease after LR therapy, $80 \%$ after primary resection and $71 \%$ after salvage chemotherapy with subsequent PC-RTR. Five patients (23\%) have been relapse-free after LR therapy (mean FU 62 months), three patients after primary resection and two after salvage chemotherapy. Ten patients died of disease during FU (45.5\%) and had a mean time from LR to death of 66 months, six patients after primary resection (40\%) and five after salvage chemotherapy (57\%). Eleven patients were alive at last FU (mean FU 62.2 months), eight patients after primary resection $(53 \%)$ and three after salvage chemotherapy (43\%).

Patients who presented viable cancer (embryonal carcinoma, yolk sac and seminoma) or somatic-type malignant transformation had a relapse rate of $90 \%$ (9/10) and $80 \%$ $(4 / 5)$, respectively, while patients who presented post-pubertal teratoma had a relapse rate of $57 \%$ (4/7). Survival rate of patients with viable cancer and somatic-type malignant transformation was 33\% (6/9) and 60\% (2/5), respectively. Teratoma patients had a more favorable survival rate at $71 \%$ $(5 / 7)$.

There was no significant difference in preoperative AFP levels between patients who suffered a relapse (mean AFP $926 \mu \mathrm{g} / \mathrm{l}$ ) and patients who were relapse-free (mean AFP $1181 \mu \mathrm{g} / \mathrm{l}$ ) after LR therapy (two-tailed $t$ test, $p=0.84$ ). AFP levels did not differ significantly either for patients who have died of disease (mean AFP of patients who died $1437 \mu \mathrm{g} / \mathrm{l}$, mean AFP for patients who are still alive $577 \mu \mathrm{g} / \mathrm{l}$, two-tailed $t$ test, $p=0.34$ ).

\section{Discussion}

Comparing patients with primary resection of LR-GCT to those who received salvage chemotherapy with subsequent PC-RTR showed no significant difference in relapse rate or survival $(p=0.66)$. The poor prognosis of late relapsing tumors has been stated in numerous previous reports $[1,3$, 4].

Patients who suffered from disease progression or a relapse after LR therapy and died of disease had a prolonged course from LR until death ( $>5$ years). This suggests that LR-GCT with elevated STMs are in most cases slowly proliferating tumors and resistant to salvage therapy. The level of AFP-elevation was not predictive of outcome or histological type of a LR-GCT regardless of LR therapy. Except choriocarcinoma, all types of malignant GCT were present in our histological findings with a high incidence of somatic-type malignant transformation (24\%). This proves the heterogeneous nature of late relapsing tumors.

Previous studies have found that patients with teratoma at the time of LR had a better outcome compared to patients presenting with viable cancer $[2,8,9]$. In our series, patients with teratoma had a $71 \%$ cancer-specific survival if somatictype malignant transformation was not present. Patients who presented with viable cancer had a considerably lower survival rate at $33 \%$.

Oldenburg et al. reported about the incidence of marker elevation in LR tumors: AFP was elevated in $49 \%$ and B-HCG in $24 \%$ of the cases [1]. Thirty-five of 37 of our patients had an AFP elevation, while only two patients

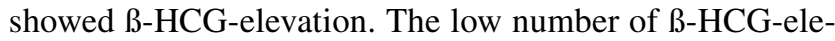
vated LR tumors in our cohort is probably attributed to bias. In our database, we only record patients who underwent surgery either as primary treatment or as secondary resection after chemotherapy. Choriocarcinoma is generally a chemosensitive tumor and, thus, underrepresented in patients with LR [10].

A remarkable finding is that although teratoma is normally characterized by normal STM [11], we found AFP elevation in 16 teratoma patients. While seven teratoma patients also presented somatic-type malignancy components, two patients with somatic-type malignancy did not show teratoma components. Tu et al. reported that patients with yolk sac-seminoma in primary histology were predisposed to undergo somatic transformation [15]. We could not observe this association of primary histology with somatic transformation in LR (see Table 3). Although a 
Table 3 Comparison of primary and late relapse histology for each patient

\begin{tabular}{|c|c|c|}
\hline Patient nr. & Primary histology & Late relapse histology \\
\hline 1 & Seminoma, teratoma & Teratoma \\
\hline 2 & $\mathrm{EC}$ & $\mathrm{EC}$ \\
\hline 3 & EC, teratoma & Teratoma \\
\hline 4 & EC & YS, teratoma \\
\hline 5 & EC & Teratoma \\
\hline 6 & EC & EC, SMT \\
\hline 7 & EC, YS & YS \\
\hline 8 & Seminoma, EC & Teratoma \\
\hline 9 & $\mathrm{CC}$ & Seminoma \\
\hline 10 & Unknown (NSGCT) & Teratoma, SMT \\
\hline 11 & Teratoma & YS \\
\hline 12 & EC, teratoma & EC \\
\hline 13 & EC & YS \\
\hline 14 & EC & EC, YS, teratoma \\
\hline 15 & EC & YS \\
\hline 16 & Seminoma & Teratoma, SMT \\
\hline 17 & Unknown (NSGCT) & EC \\
\hline 18 & EC, teratoma & EC, teratoma \\
\hline 19 & $\mathrm{CC}$ & YS, teratoma \\
\hline 20 & Teratoma & Teratoma \\
\hline 21 & Unknown (NSGCT) & Teratoma \\
\hline 22 & Unknown (NSGCT) & Teratoma \\
\hline 23 & Seminoma & YS \\
\hline 24 & CC, teratoma & Teratoma \\
\hline 25 & Seminoma, teratoma, adenocarcinoma, sarcoma & SMT \\
\hline 26 & Teratoma & Teratoma, SMT \\
\hline 27 & NSGCT & YS, seminoma \\
\hline 28 & YS, CC, teratoma & Teratoma \\
\hline 29 & Unknown (NSGCT) & YS, teratoma \\
\hline 30 & Teratoma & EC, teratoma \\
\hline 31 & EC & Teratoma \\
\hline 32 & $\mathrm{CC}$, teratoma & Teratoma, SMT \\
\hline 33 & Seminoma & Teratoma, SMT \\
\hline 34 & EC, teratoma & EC, teratoma \\
\hline 35 & EC, YS, CC & EC \\
\hline 36 & EC, teratoma & Teratoma, SMT \\
\hline 37 & Seminoma, EC & Teratoma, SMT \\
\hline
\end{tabular}

In total, $54 \%$ of patients' primary histology differs from late relapse histology (highlighted)

NSGCT non-seminomatous germ cell tumor, EC embryonal carcinoma, YS yolk sac, CC choriocarcinoma, SMT somatic-type malignant transformation 
Table 4 Outcome comparison of therapy groups

\begin{tabular}{|c|c|c|c|c|c|c|}
\hline & Relapse & $\mathrm{n}=$ & $\%$ & Dead & & $\%$ \\
\hline \hline Surgery & yes & 12 & $80 \%$ & yes & 6 & $43 \%$ \\
\hline & no & 3 & $20 \%$ & no & 8 & $57 \%$ \\
\hline \hline Chemotherapy & yes & 5 & $71 \%$ & yes & 4 & $57 \%$ \\
\hline & no & 2 & $29 \%$ & no & 3 & $43 \%$ \\
\hline
\end{tabular}

low level of AFP-elevation can be due to non-neoplastic reasons, four teratoma patients showed very high AFP levels $(135,281,365$ and $2533 \mu \mathrm{g} / \mathrm{l})$. As AFP has been used as a marker of endodermal stem cell differentiation [12], we hypothesize that AFP could have been induced during the endodermal differentiation of an embryonal carcinoma into the three germ layers (teratoma). Alternatively, the AFP expression might have originated from undetected yolk sac tumor cells. This hypothesis may be applied even more so to those presenting with somatic-type malignancy because sarcomatous somatic-type malignancy has been reported to derive from yolk sac tumor lineage [13, 14].

A major limitation of this study is that the indication for primary resection or chemotherapy was based on individual considerations. Some patients were referred to our center for post-chemotherapy surgery after undergoing chemotherapy in another center. Thus, patients with a priori resectable findings underwent surgery, while patients with non-resectable findings, such as bone or hepatic metastases, and therefore worse prognosis per se, received chemotherapy. There is also a clear difference in the median STM levels before primary LR resection or chemotherapy (Table $1, t$ test, two-tailed, $p=<0.0001$ ). A second major limitation is the high percentage of patients who were lost to follow-up. This fact reduces the validity of our follow-up analysis.

Nevertheless, we could show that in up to $54 \%$ of the cases the primary histology did not coincide with the findings on LR histology. On the one hand, patients with teratoma in the LR specimen in particular benefit from surgical treatment, as chemotherapy is not effective for this type of germ cell tumor. In our series, even some pure teratomas and somatic-type malignancies showed highly elevated STMs before surgery (see Table 2). Therefore, our recommended strategy is to perform primary surgery regardless of STM level if lesions are resectable to exclude pure teratoma or somatic-type malignancies in order to prevent unnecessary chemotherapy. On the other hand, even patients with completely resected LR had an unexpectedly high recurrence and, consecutively, a low cancer-specific survival rate. This new information on the outcome of patients with completely resected LR and elevated markers should guide the shared decision making of treatment and ask for close follow-up with early systemic salvage treatment if possible.

\section{Conclusions}

Patients with a late relapsing germ cell tumor and elevated markers have an unexpectedly poor prognosis and a high risk for another relapse, independent of treatment, even if completely resected with primary surgery. The histological type and aggressiveness of a late relapsing tumor cannot be predicted with serum tumor marker levels at the time of diagnosis of LR. In up to $54 \%$ of cases, primary histology did not coincide with LR histology. Therefore, we propose primary surgical resection of a late relapsing tumor with elevated markers if a complete resection is feasible in order to prevent unnecessary chemotherapy in patients with teratoma and somatic-type malignancies. Since every second patient even with complete resection and marker normalization experiences relapse and faces a poor survival, novel systemic treatment options need to be developed.

Author contributions YC helped in conception, writing the manuscript, first draft, data analysis, implementation of review, final draft. $\mathrm{AL}$ acquired the data and critically reviewed the manuscript. Winter acquired the data and critically reviewed the manuscript. CB managed and analyzed the data and critically reviewed the manuscript. RGS acquired and analyzed the data and critically reviewed the manuscript. PA contributed to conception, data acquisition, critical review. AH helped in conception/idea, data management, review of data and analysis, and final draft.

Funding Open Access funding enabled and organized by Projekt DEAL.

\section{Declarations}

Conflict of Interest The authors indicate no potential conflicts of interest.

Research involving human participants Ethical approval was waived by the Local Ethics Committee of University of Düsseldorf (HeinrichHeine University Duesseldorf) in view of the retrospective nature of 
the study, and all the procedures being performed were part of the routine care. This study was performed in line with the principles of the Declaration of Helsinki.

Informed consent All patients have signed a written informed consent form and gave their approval for this study. All data presented have been anonymized.

Disclaimer We confirm that the manuscript has been read and approved by all named authors and that there are no other persons who satisfied the criteria for authorship but are not listed.

Open Access This article is licensed under a Creative Commons Attribution 4.0 International License, which permits use, sharing, adaptation, distribution and reproduction in any medium or format, as long as you give appropriate credit to the original author(s) and the source, provide a link to the Creative Commons licence, and indicate if changes were made. The images or other third party material in this article are included in the article's Creative Commons licence, unless indicated otherwise in a credit line to the material. If material is not included in the article's Creative Commons licence and your intended use is not permitted by statutory regulation or exceeds the permitted use, you will need to obtain permission directly from the copyright holder. To view a copy of this licence, visit http://creativecommons.org/licenses/by/4.0/.

\section{References}

1. Oldenburg J, Martin JM, Fosså SD (2006) Late relapses of germ cell malignancies: incidence, management, and prognosis. J Clin Oncol 24(35):5503-5511

2. Michael H, Lucia J, Foster RS, Ulbright TM (2000) The pathology of late recurrence of testicular germ cell tumors. Am J Surg Pathol 24(2):257-273. https://doi.org/10.1097/00000478-20000 2000-00012

3. Lipphardt ME, Albers P (2004) Late relapse of testicular cancer. World J Urol 22(1):47-54

4. O'Shaughnessy MJ, Feldman DR, Carver BS, Sheinfeld J (2015) Late relapse of testicular germ cell tumors. Urol Cancer 42(3):359-368

5. Baniel J, Foster RS, Gonin R, Messemer JE, Donohue JP, Einhorn LH (1995) Late relapse of testicular cancer. J Clin Oncol 13(5):1170-1176
6. International Germ Cell Cancer Collaborative Group (1997) International germ cell consensus classification: a prognostic factor-based staging system for metastatic germ cell cancers. J Clin Oncol 15(2):594-603

7. Ehrlich Y, Beck SDW, Foster RS, Bihrle R, Einhorn LH (2013) Serum tumor markers in testicular cancer. Urol Oncol 31(1):17-23

8. Oldenburg J, Alfsen GC, Wæhre H, Fosså SD (2006) Late recurrences of germ cell malignancies: a population-based experience over three decades. Br J Cancer 94(6):820-827

9. Sharp DS, Carver BS, Eggener SE, Kondagunta GV, Motzer RJ, Bosl GJ et al (2008) Clinical outcome and predictors of survival in late relapse of germ cell tumor. J Clin Oncol 26(34):5524-5529

10. Speir RW, Calaway AC, Einhorn LH, Foster RS, Cary C (2020) Postchemotherapy retroperitoneal lymph node dissection in patients presenting with very high HCG levels. Urol Oncol Semin Orig Investig 38(8):687.e19-687.e23

11. Talerman A, Haije WG, Baggerman L (1980) Serum alphafetoprotein (AFP) in patients with germ cell tumors of the gonads and extragonadal sites: correlation between endodermal sinus (yolk sac) tumor and raised serum AFP. Cancer 46(2):380-385

12. Gualdi R, Bossard P, Zheng M, Hamada Y, Coleman JR, Zaret KS (1996) Hepatic specification of the gut endoderm in vitro: cell signaling and transcriptional control. Genes Dev 10(13):1670-1682

13. Howitt BE, Magers MJ, Rice KR, Cole CD, Ulbright TM (2015) Many postchemotherapy sarcomatous tumors in patients with testicular germ cell tumors are sarcomatoid yolk sac tumors: a study of 33 cases. Am J Surg Pathol 39(2):251-259. https://doi.org/10. 1097/PAS.0000000000000322

14. Williamson SR, Delahunt B, Magi-Galluzzi C, Algaba F, Egevad L, Ulbright TM et al (2017) The World Health Organization 2016 classification of testicular germ cell tumours: a review and update from the International Society of Urological Pathology Testis Consultation Panel. Histopathology 70(3):335-346

15. Tu S-M, Bilen MA, Hess KR, Broaddus RR, Kopetz S, Wei C et al (2016) Intratumoral heterogeneity: role of differentiation in a potentially lethal phenotype of testicular cancer. Cancer 122(12):1836-1843

Publisher's Note Springer Nature remains neutral with regard to jurisdictional claims in published maps and institutional affiliations. 